\title{
Nomenclature and Significance of Innate/Natural Immune Mechanisms and of Species Specific Resistance
}

\author{
Lóránd Bertók ${ }^{\mathrm{a}, *}$ and István Berczi ${ }^{\mathrm{b}, \mathrm{c}}$ \\ ${ }^{a}$ Frédéric Joliot-Curie" National Research Institute of Radiobiology and Radio Hygiene, Budapest, Hungary \\ ${ }^{\mathrm{b}}$ Department of Immunology, Faculty of Medicine, The University of Manitoba, Winnipeg, MB, Canada \\ ${ }^{\mathrm{c}}$ Department of Physiology, Free University of Augascalientes, Augascalientes, Mexico
}

\begin{abstract}
The immune system of higher animals comprised of two parts: Innate - or Natural - (INIM-NIM) and Adaptive Immunity $(A D I M)$. Innate resistance includes physical barriers (e.g. skin, cornea, mucous membranes, the blood-brain barrier $\mathrm{BBB}$ ) chemical defense ( $\mathrm{HCl}$ in the stomach, bile acids in the intestines, enzyme based protection) and Species Specific Resistance (SSR) to viruses and other pathogens. SSR may occur because of the lack of proper receptors for the pathogen to establish itself in the resistant animal species. Therefore, SSR is a phylogenetically determined property of various animal species. Innate immunity includes polyspecifc immune mechanisms with germ line gene based antigen receptors (INIR/NIR) that recognize phylogenetically preserved, highly cross-reactive (homologous) epitopes, or homotopes. Because both the antigen receptors (e.g. toll-like receptors - TLR) and the homotopes (e.g., lipid A in bacterial lipopolysaccharide - LPS) are constant, and fully differentiated immune (monocyte/macrophages and other leucocytes) and somatic cells express TLR, the entire organism is capable of responding instantaneously to infection or injury. Innate phagocytes, primarily monocyte/macrophages, support the development of adaptive immunity, by presenting antigen and by contributing cytokines for cell activation. If natural immunity is depressed, ADIM will be deficient. The natural immune system provides continuous protection for the host animals or men against the effects of stress, treatment of cytostatic drugs, septic shock, infectious diseases, and numerous other noxious agents.

Neuroendocrine, nutritional and environmental factors regulate natural immunity. The maintenance of good NIM defense is most important in modern medicine. Radiodetoxified endotoxin (RD-LPS) stimulates NIM, and activates the bone marrow. Animal experiments and human studies showed that RD-LPS is a promising preparation for the elevation of natural immunity. RD-LPS is a potent immunological adjuvant in the case of inactivated virus vaccines (e.g. influenza).
\end{abstract}

\section{HISTORY}

Fever has been regarded in Persia and in aintient Egypt as a healing reaction. This beleif survived until 1933, when Boivin [1] purified bacterial lipopolysaccharide (LPS) for the purposes of fever therapy. LPS is a very effective pyrogen. The biological effects of

\footnotetext{
*Correspondence to: Lóránd Bertók, DVM, Ph.D., National Research Institute for Radiobiology and Radiohygiene, 1221 Budapest, Anna u.5, (H-1775 Budapest, P.O. Box 101), Hungary. Tel.: +36 1/229 1956; Fax: +36 1/229 1956; E-mail: bertok@ osski.hu.
}

LPS has been investigated with keen interst and in 1983 Nowotny summarised the beneficial effects of endotoxin [2].

At the beginning of the last century, Wright [3] was among the first to study the natural immune resistance. He observed that after vaccination with killed bacteria a 'negative' phase occurred, and an increase in natural resistance followed, which was tied to the production of specific antibodies. Landy and Pillemer [4] and Rowley [5] made similar observations later independently and demonstrated that this pre-immune resistance was due to the LPS content of the vaccines 
used. These authors also observed that such changes in immune status stimulated the production of natural antibodies [6]. Pillemer et al. [7] discovered the properdin system. LPS stimulated properdin. Beeson [8] discovered that low doses of toxic LPS given repeatedly to animals lead to the induction of endotoxin tolerance, which prevented also the pyrogenic effect of LPS (pyrogen tolerance). Small LPS doses decreased the severity of various forms of experimental shock and of the lethal effect of radiation. Later it was demonstrated that LPS functioned as an immunological adjuvant, and it was capable of inducing tumor necrosis. LPS also stimulated bone marrow activity and increased interferon production. Moreover, infectious diseases were prevented by small LPS doses, which significantly increased natural resistance. These observations have evoked great interest in the phenomenon of endotoxin tolerance [9].

\section{INNATE/NATURAL HOST DFENSE}

The subdivision of the Immune System to various forms of immunity/resistance are presented in Fig. 1.

\section{Non-immune resistance}

\section{Species specific resistance}

Numerous examples are there for species specific resistance to infectious agents. If one consults a book on Veterinary Microbiology of Infectious Diseases, he/she will find numerous bacteria and viruses, protozoa and fungi that show species specificity. Species specific diseases are the rule, polyspecific agents are the exception. A good example is Mycobacteia. We are aware of the human pathogen (M. tuberculosis), the bovine pathogen (M. bovis), and pathogenic strain

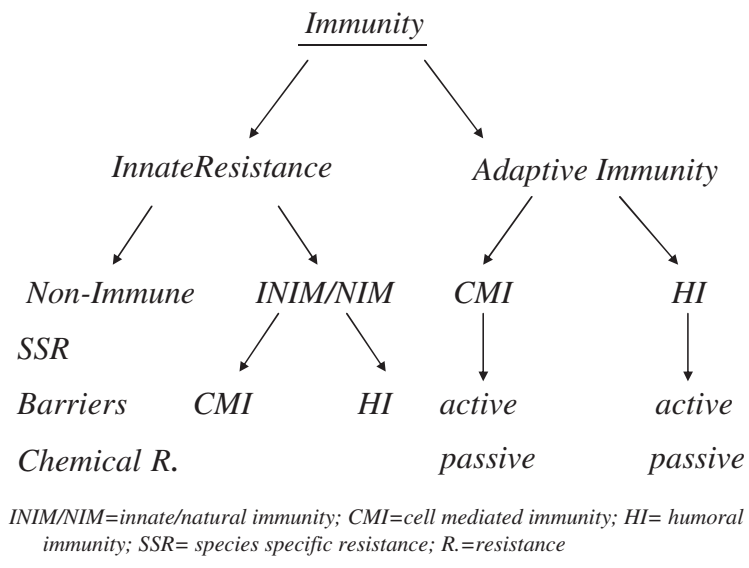

Fig. 1. Subdivision of the immune system. for birds (M. avium). Today it is clear that infectious agents use specific receptors, which make their persistence possible in the host organism. If such receptors are missing, no persistence for the infectious agent is possible.

\section{Barriers in host defense}

It is of common knowledge that the skin functions as a mechanical barrier to the penetration of microorganisms and other noxious agents into the body. Lister [9] recognized first the significance of sterile environment in surgery (e.g. used carbolic acid to sterilize) without the knowledge of pathogenic microbes. Pasteur [9] and Koch [9], discovered pathogenic microbes, which knowledge gave scientific support for sterility in surgery. Today it is a fundamental rule in surgery that wounds are maintained under sterile conditions. The skin also has an elaborate immune system, with Langerhans cell presenting antigen, $\mathrm{T}$ and $\mathrm{B}$ cells and cells of innate immunity, such as macrophages and NK cells, which complements the mechanical defense with highly efficient immune protection [10]. Neuroimmune mechanisms regulate immune function in the skin [11].

Mucous membranes also function as mechanical barriers, however these membranes are protected very intensively by secretory IgA antibodies, and also cellular elements of the immune system (phagocytic cells, $\mathrm{T}$ and $\mathrm{B}$ lymphocytes, macrophages and dendritic cells presenting antigen). The Payer patches and mesenteric lymph nodes are the centers of mucosal immunity in the gut. Cytokines, chemokines, defensins, all play a role in the mucosal defense system [12].

The alimentary tract is exposed continuously to food antigens. Such massive stimulation would rapidly exhaust the capacity of the immune system. Therefore, the induction of immunological tolerance is of vital importance to food antigens in the gastrointestinal tract. The immunosuppressive enzyme, kallikrein, and $\mathrm{T}$ suppressor cells producing transforming growth factor (TGF)- $\beta$ generate immunological tolerance to food antigens. Infectious agents invariably penetrate the gut mucosa or even deeper, where they are handled by the host's immune system. Large amount of kallikrein is produced in the submandibular gland. Production is regulated by, androgens, prolactin, glucocorticoids, and thyroxin [13].

\section{Barrier systems of the brain}

The CNS is separated from the blood by the blood by the $\mathrm{BBB}$, which is localized at the level of the 
endothelial cells and separates brain interstitial fluid (ISF) from blood; at the epithelial layer of four choroid plexuses, the blood-cerebrospinal fluid (CSF) barrier (BCSFB), which separates CSF from the CP epithelial cell - ISF, and at the arachnoid barrier [14].

The most sophisticted barrier sytem in the body is the BBB. While the thight endothelial junctions are fundamental to the efficiency of the barrier, this is supplemented with multiple regulatory mechanisms to regulate the entry of nutrients, minerals and immune globulins and cells. BBB is a selective permeable barrier that isolates the central nervous system (CNS) from the blood. This homeostatic system is regulated by a complex network of cellular and extracellular components. These allow the unidirectional transport of molecules through the vascular endothelial cells by an asymmetrical distribution of specific receptormediated transport proteins and selective ion channels located on the luminal and abluminal faces. The polarized transport is primarily due to the presence of tight junctions between perivascular endothelial cells, yet accessory cells provide the needed signals for differentiation and maintenance of the endothelium. Without the influence of these cells, the barrier mechanisms would not be formed and blood-borne cells would not be excluded. The BBB express limited number of adhesion molecules by the endothelial cells and there is a paucity of released chemokine signals. These factors contribute to the effectiveness of BBB. By now the idea that the BBB isolates the brain from the immune system as a privileged organ has been largely abandoned. Even under homeostasis, the BBB is able to facilitate the entry of infectious pathogens and antibodies into the CNS by receptor-mediated transport. The BBB also provides discrete transmigration pathways for immune cells to continuously patrol the CNS. In the setting of inflammation, BBB cells are active participants that permit the development of routes for antibody and immune cell extravasation, immune recognition, and augmentation of the inflammatory response [15].

In the brain the pericyte is an important cellular component of the BBB and, together with other cells of the neurovascular unit (endothelial cells, astrocytes and neurons) the pericyte makes fine-tuned regulatory adjustments and adaptations to promote tissue survival. These regulatory changes involve trans-cellular communication networks between cells. [16].

Bidirectional crosstalk between the immune cell and endothelium is an essential element in diapedesis during both normal immune surveillance and under inflammatory conditions. The signalling networks are induced through engagement of cell surface molecules such as integrins on the leucocyte and immunoglobulin superfamily cell adhesion molecules on the endothelial cell, which play a major role in determining the pattern and route of leucocyte emigration [17].

\section{Chemical host defense}

We observed in piglets that parenterally applied LPS isolated from pathogenic E. coli organisms elicited a disease, which clinically and pathologically was identical to the disease induced by the pathogenic E. coli bacteria. Thus, LPS was designated as the pathogenic principle of virulent strains of $E$. coli. Such pathogenic bacteria usually caused severe diarrhea, fever, apathy, and severe fluid loss, which often had fatal outcome [18].

LPS injected rats developed endotoxin shock, which was accompanied by fever, loss of appetite, diarrhea and in severe cases, death. However, such intoxication was absent after oral application of LPS to rats, not even after oral treatment jointly with compound $48 / 80$, which is a mast cell discharger and caused injury to the intestinal tract [19].

In order for LPS to have a pathogenic effect orally it was necessary to ligate the bile duct. The absence of bile made rats sensitive to the oral application of LPS. Subsequently it was determined that bile acids, which are known the act as detergents, could degrade LPS and thus neutralize its toxic activity. Therefore, bile acids in the gastrointestinal tract protect the host from intoxication by LPS released from intestinal Gram-negative bacteria. However, bile acids are not produced in cases of some severe diseases (e.g. hemorrhagic trauma, radiation disease, sepsis, hepato- renal syndrome, Parvovirus infection, herpes, psoriasis, atherosclerosis, etc) and consequently, LPS absorbs from the gut. These conditions can be prevented or treated with bile acids. In cases of moderate absorption LPS stimulates the natural immune defense and bone marrow function of the host, which helps recovery [20].

Acid mediated resistance in the stomach. In the stomach $\mathrm{HCl}$ is secreted, which maintains the $\mathrm{PH}$ around 2, which is very acidic. Such acidity would kill most microorganisms, including pathogenic organisms. That acid environment inhibit microbes is well known as pickling is a well recognized method of food preservation. Acetic acid is useful as a preserving agent. Obviously, the acidity of the stomach is serving as a chemical barrier to many infectious agents that enter the digestive tract. Therefore, chemical barrier represents one form of natural resistance to infectious agents. However, some infectious agents are able to 
cope with this hostile environment (e.g., Helicobacter pylori, that causes ulcers in the stomach).

Enzymatic defence. As it is discussed in Physiology books, one of the major functions of the liver is to detoxify the blood, primarily by enzymatic degradation. The liver is capable of "adaptive enzyme synthesis" the function is to make the detoxifying process more efficient. A human disease is characterized by the extreme enlargement of the colon (megacolon). These people fall ill, apparently because the liver is not able to detoxify all the noxious agents that absorb from the huge colon [9].

The liver is also capable of producing acute phase proteins (APP), which are enzyme inhibitors, recognize molecules of pathogenic agents (CRP, EBP, MBP), complement, fibrinogen etc. The liver is a vey important organ in natural immunity [21].

\section{Natural immune host defense}

The natural immune system provides defense instantaneously when infection/insult occur. Germ line genes code for INIR, and these receptors are constant, never change. INIR is present on all leukocytes and possibly on most cells and organs in the body. Recently TLR were found in numerous tissues and organs (see below). Fully differentiated cells express INIR and such cells are able to respond instantaneously when stimulated by infections or by other pathogenic agents. INIR recognize evolutionarily conserved and highly cross-reactive (homologous) epitopes, or homotopes. For instance, lipid A is a homotope, which is present in bacterial LPS and is recognized by TLR4. LPS is expressed in all species and serotypes of Gram negative bacteria, regardless of pathogenesis. LPS stores sugar, which is required for DNA synthesis in Gram negative organisms, so it is necessary for survival. LPS negative mutants of Gram negative bacteria are not viable. This makes LPS an ideal target for INIR. It is cross-reactive and must be expressed by all members of the target organisms, and no mutations are possible. Therefore, TLR4 is able to protect against Gram negative infections, no matter what strain or serotype is involved [22-24]. Others call innate immune recognition as "pattern recognition" [24].

\section{INIR properties and distribution in the body}

Phosphoryl choline, galactose and cationic proteins are homotopes present in microbes and in degraded cells. The acute phase protein (APP), $C$ reactive protein $(\mathrm{CRP})$, recognize these homotopes and subsequently may activate $\mathrm{T}$ and B lymphocytes, macrophages, the complement system, polymorphonuclear phagocytes, natural killer (NK) cells and platelets. Mannose binding proteins $(\mathrm{MBP})$ are also APPs and recognize diverse carbohydrates, which are present in microbes. MBP activates macrophages, polymorphonuclear cells and the complement system. Natural antibodies recognize diverse homotopes, such as charged molecules, carbohydrates and superantigens. These homotopes are present in microbes, cancer cells and in self components. B and T cells, macrophages, complement, polymorphonuclear - and NK cells are activated by natural antibodies. Superantigens (microbial toxins) are present in microbes and self components. Act on the T and B cell receptors and are capable of polyclonal activation. In severe cases the cytokines released as the consequence of polyclonal lymphocyte activation may kill the host organism. Lipid, glycolipid, peptides are present in microbes and may be present in self-antigens. T lymphocytes recognize these homotopes by their CD1 surface receptors. Phosphate containing nonpeptides are present in microbes and may be present in selfantigens. These are recognized by $\gamma / \delta \mathrm{T}$ lymphocytes with their specific receptors [22-24].

\section{TLR in host defense}

As already mentioned, lipid $\mathrm{A}$ is one homotope expressed in LPS of Gram negative bacteria. In the serum of animals and of man an APP is present during acute illness called LPS binding protein (LBP). LBP binds LPS in the serum and then combined with the cell surface receptor, CD14, which is present on B cells, macrophages, platelets, endothelium, the central nervous system and endocrine glands. CD14 will then interact with TLR4, which activates nuclear factor kappa B $(\mathrm{NF}-\kappa \mathrm{B})$ in the cells so stimulated. Such activation induces cytokines, which initiate a defense reaction against the infection [21, 22, 25, 26].

TLR1/TLR2 recognize bacteria, Neisseria; and the homotopes being $\mathrm{Pam}_{3}$ Cys lipopeptides/lipoproteins, soluble factor. TLR2/TLR6 recognize mycoplasma (Pam 2 Cys Lipopetides (MALP-2); yeast (zymosan), Gram positive bacteria (peptideglycan), and Staphylococcus (modulin). TLR2/TLRX recognize Gram positive bacteria (Lipoteichoic acid); Gram negative bacteria (capsular polysaccharide); Leptospira (lipopolisaccharide); Mycobacteria (lipoarabinomannan, glycolipids); measeless virus (hemagglutinin protein); Protozoa (Glycosyl-phosphatidyl inositol ancors); Spirochetes (Glycolipids). TLR3 recognizes viruses (ds DNA). TLR4 recognizes Gram negative 


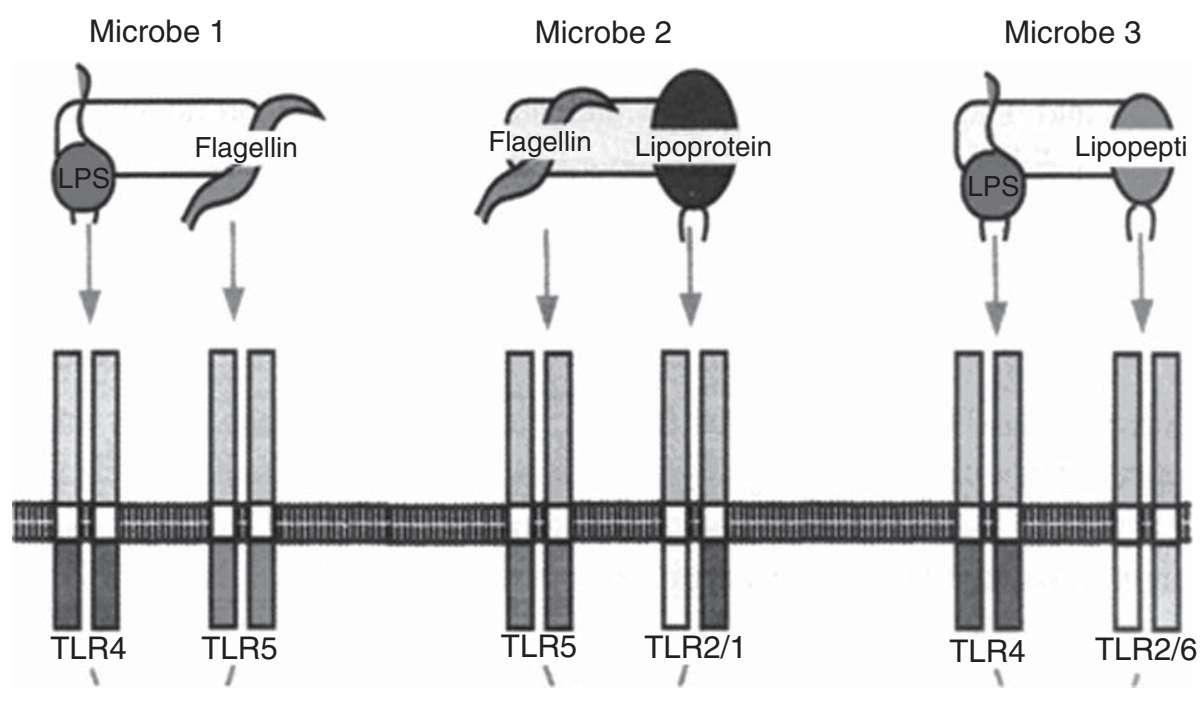

Fig. 2. Homotope recognition by TLR. Modified from reference [23].

bacteria (LPS, capsular polysaccharide); Mycobacterium tuberculosis (heat labile cell associated factor); CMV virus, murine retrovirus (F-protein, envelope protein). TLR5 recognizes bacteria (Flagellin). TLR7/TLR8 target is not known (bind imidazoquinolines). TLR 9 recognizes bacteria (unmethylated $\mathrm{CpG}$ DNA) (Fig. 2) [26].

$T L R$ signaling. TLRI/2 are stimulated by lipoproteins and lipopeptides - activate nuclear factor $-\kappa \mathrm{B}$ $(\mathrm{NF}-\kappa \mathrm{B})$ in the nucleus, which is followed by the stimulation of TNF- $\alpha$ and IL- 6 secretion and dendritic cell (DC) maturation. TLR3 is activated by ds RNA, activates interferon regulatory factor 3 (IRF)-3, and stimulates IFN- $\beta$ and IP10, a nuclear regulatory protein. TLR4 is activated by LPS, stimulates NF- $\kappa \mathrm{B}$ and promotes DC maturation. TLR 7/8 recognize imida-

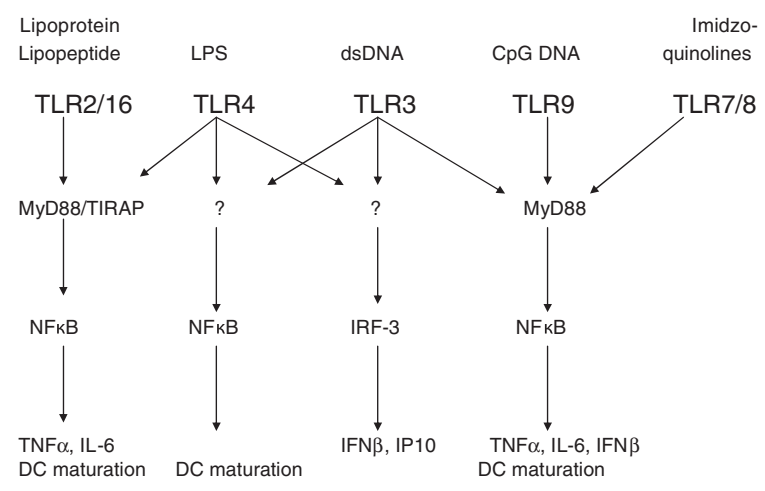

Fig. 3. TLR Signaling. Modified from reference [23]. zoquinoline and TLR9 recognize CpG DNA, activate NF- $\kappa$ B and stimulate TNF- $\alpha$, IL6, INF- $\beta$ and DC maturation (Fig. 3) [26].

\section{TLR distribution in the body}

Because the BBB protects the CNS from invasion by immune/inflammatory cells an even from macromolecules [14-17], the brain relays heavily on its INIM system for defense during homeostasis. It appears that all cells in the brain, including neurons and nerves express innate immune receptors, and are capable of responding to homotopes, which are recognized by INIR. Within the CNS the chorid plexus participates in acute phase responses and is able to produce acute phase proteins (APP). If, however, this system is incapable of handling the insult, there are mechanisms to admit the cells of adaptive immunity (e.g. macrophages, $\mathrm{T}$ and $\mathrm{B}$ cells other leukocytes) into the CNS.

All leukocytes express TLR. TLR are expressed in the pituitary gland, in the adrenal gland, in the liver in mucosal epithelial cells, in endothelial cells, in vascular smooth muscle and also in the cornea (Table 1) [23].

These facts suggest that the entire body, including the CNS, endocrine organs, the liver, epithelium and endothelium, and possibly even more, participate in innate immune host defense. The CNS is capable of directly sensing infectious agents trough TLR, and possibly by other INIR, and react instantaneously by causing inflammation, the final effector response of 
Table 1

Biological effects of LPS, C-reactive protein and endotoxin binding protein [modified from reference 22]

\begin{tabular}{|c|c|c|c|c|}
\hline & Biol. Effect & LPS+/- LBP & CRP & $\mathrm{NAb}$ \\
\hline 1 & Complement act. & + & + & + \\
\hline 2. & $\begin{array}{l}\text { Monocyte/macrof. } \\
\text { Kupfer cells, liver. }\end{array}$ & + & + & \\
\hline 3. & $\mathrm{~T}$ cells & $+\mathrm{T}(»)$ & $+(\mathrm{CTL})$ & + \\
\hline 4. & $\mathrm{~B}$ cells & + & + & + \\
\hline 5. & Mast cells & $+($ via C3a, C5a $)$ & $+($ via C3a, C5a) & + \\
\hline 6. & $\begin{array}{l}\text { Polymorphonuclear } \\
\text { cells }\end{array}$ & $+($ via C3a, C5a) & $+($ via C3a, C5a) & + \\
\hline 7. & NK cells & - & + & + \\
\hline 8. & Platelets & + & + & $+?$ \\
\hline 9. & Endothelium & + & & \\
\hline 10 & Peritoneum & + & & \\
\hline 11. & Smooth muscle & + & & \\
\hline 12 & Cornea & + & & \\
\hline 13 & CNS (neurons, glia) & + & & \\
\hline & $\begin{array}{l}\text { Vagus and sensory } \\
\text { nerves }\end{array}$ & + & & \\
\hline 15 & $\begin{array}{l}\text { Endocrine glands } \\
\text { (paren- chimal cells, } \\
\text { macrophages) }\end{array}$ & + & & \\
\hline
\end{tabular}

lps = Lipopolysaccharide; $\mathrm{LBP}=\mathrm{LPS}$ binding protein; $\mathrm{NAb}=$ Natural antibody; $\mathrm{T}$ cell $=$ thymus-derived lymphocyte; $\mathrm{CTL}=$ cytotoxic $\mathrm{T}$ lymphocyte; $\mathrm{B}$ cells = bone marrow derived lymphocytes; C3a, C5a = complement split products, "anapylatoxins"; NK cells = natural killer cells; $\mathrm{CNS}=$ central nervous system; +, biological effect is present; -, biological effect is absent.

all immune compartments. Similarly the pituitary produces POMC in response to LPS (TLR4 is involved), mucosal epithelial TLR participate in inflammation and respond to pathogens, corneal TLR was found to fight infection and endothelial TLR was observed to play important roles in homeostasis of the heart. Therefore, in addition to participating in INIM, TLR fulfill important physiological functions. This is true for cytokines and also for cellular elements of the immune system, whether or not natural or adaptive immune cells are considered (Table 1) [23].

\section{Mast cells as sensory organs of the CNS}

During homeostasis the cytokine level in the blood is too low to exert feedback signals directly to the brain. Therefore, sensory nerves, which sense locally cytokine signals, transmit signals from immune organs and from sites of minor infections/inflammation to the CNS. The vagus nerve contains sensory fibers which forward signals to the CNS from visceral organs [20].

Must cells are in close contacct with sensory nerves, (Fig. 5) [24] and mediate neurogenic inflammation, which is a major effector arm of the Neuroimmune Supersystem [25, 26]. Therefore, must cells serve as nociceptors, and also as immunologically specific receptors capable of delivering infomation to the CNS, for approximately about $10^{11}$ epitopes. Must cells also recognize complement split products (C3a, C5a - "anaphylatoxins") and inform the brain about complement (C') fixation, as well as cause inflammation in response to $\mathrm{C}^{\prime}$ activation. Bradykinine is an inflammatory mediator, which signals the brain about inflammatory events via sensory nerves (Fig. 4) [23].

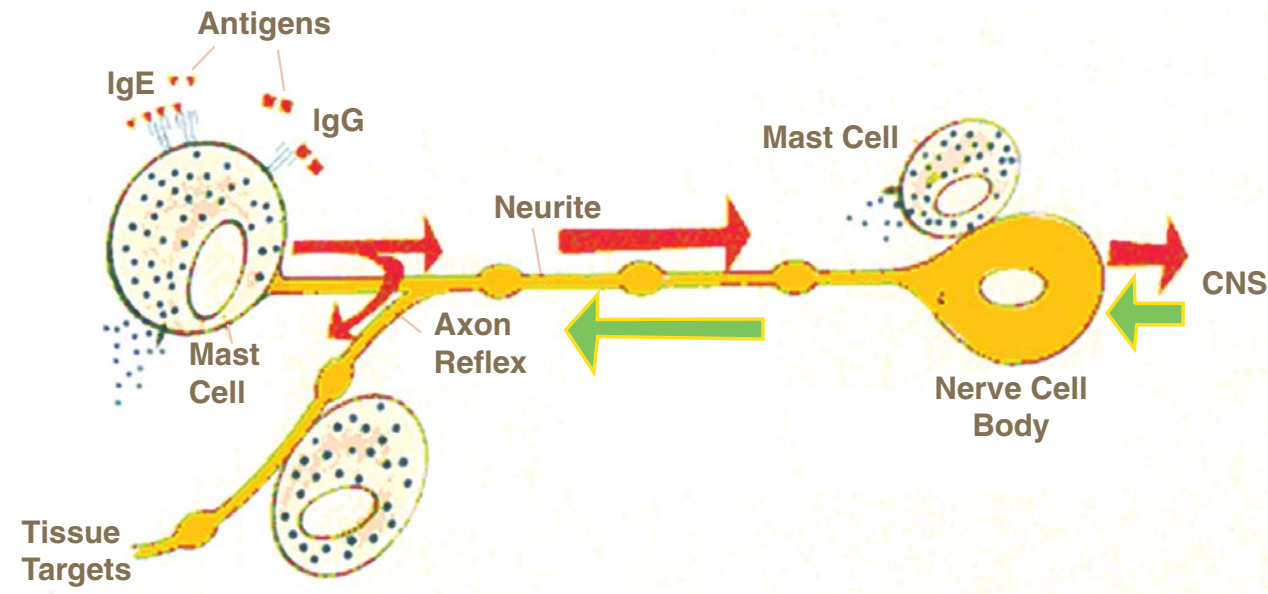

Fig. 4. Mast Cells and Neurogenic inflammation. Mast cells behave as sensory receptors for homotopes of the antigen. Passive sensitization of mast cells takes place by the combination specific antibodies (IgE or IgG) with their Fc receptors on the cell membrane. Upon subsequent interactions with allergen, mast cell will release mediators that affect nerves in the local environment. Information passes back to the nerve cell body, and affect, by axon reflex, local tissue targets. Trough interneuronal contacts, information will go to the spinal cord and/or to the central nervous system (paraventricular nucleus). Alternatively the central nervous system will act by efferent system to modulate must cell function (Modified from John Bienenstock). 
The innate immune host defence sytem

During homeostasis the brain relies on innnate immuity for defense. The BBB and other defence mechanisms, such as the Fas/Fas ligand system, keep out cells of the adaptive immune sytems as such cells may be cytotoxic or cause inflammation, which is damaging to brain tissue. However, in emergency situations there are mechnisms that allow the participatin of adaptive immune cells in the defence of the CNS under controlled conditions [14-17].

The fetus also relies on innate immunity for defense as the adaptive immune sytem matures shortly after birth. So there is no adaptive immunocopetent cells in the fetus [27].

The innate immune system is always with us, it is indestructable, becausse the cells mediating INIM can work autonomously and react directly to homotopes by their INIR. Similarly these cells express receptors to cytokines and are able to mount atunomously innate immune responses. The destruction of the PVN, hypophysectomy, neurointermediate lobectomy, aneriour pituitary lobectomy, adrenalectomy and gonadectomy do not destroy the innate immune system, it survives and keps to defened the host organism under any circumstances [29].

\section{STUDIES WITH ENDOTOXIN}

\section{Comparative toxicity of LPS}

Domesticated mammals have extreme sensitivity to LPS, laboratory rodents show more resistance, and chickens, frogs and fish are resistant (Table 2). It seems
Table 2

Comparative toxicity of endotoxin. Modified from reference [28]

\begin{tabular}{lccc}
\hline Species & $\begin{array}{c}\text { No. } \\
\text { animals }\end{array}$ & Body weight & $\begin{array}{c}\text { Lethal dose } \\
(\mathrm{mg} / \mathrm{kg})\end{array}$ \\
\hline Calf & 1 & $500 \mathrm{~kg}$ & 0.025 \\
Rabbit & 3 & $55 \pm 10 \mathrm{~kg}$ & \\
Dog & 15 & $3 \pm 1 \mathrm{~kg}$ & 3.0 \\
Swine & 6 & $10 \pm 5 \mathrm{~kg}$ & 4.0 \\
Guinea pig & 12 & $10 \pm 4 \mathrm{~kg}$ & 5.0 \\
Cat & 20 & $450 \pm 50 \mathrm{~g}$ & 10.0 \\
Rat & 6 & $2.5 \pm 0.5 \mathrm{~kg}$ & 15.0 \\
Mouse & 200 & $300 \pm 50 \mathrm{~g}$ & $20-60.0$ \\
Chicken & 400 & $18 \pm 4 \mathrm{~g}$ & $25-60.0$ \\
Frog & 30 & $1 \pm 0.2 \mathrm{~kg}$ & $>50.0$ \\
Fish (carp) & 70 & $40 \pm 10 \mathrm{~g}$ & $>100.0$ \\
\hline
\end{tabular}

apparent that LPS toxicity is proportional to the development of the immune system of the animals exposed. Animals with low immune development are resistant to intoxication [31].

Another study in adrenalectomized (ADX) mice shed light on LPS toxicity. ADX mice were $~ 500$ times more sensitive to LPS than were normal animals. Serum TNF- $\alpha$ response to LPS was 60 times higher in ADX mice, as compared with controls. Treatment of ADX mice with dexamethasone (DEX) normalized TNF levels and restored normal sensitivity to endotoxin. These experiments indicated that in ADX mice LPS over stimulated the secretion of TNF, which in turn killed the host. The inhibitory effect of DEX, which is a glucocorticoid analogue, indicates the important role of the hypothalamus-pituitary-adrenal (HPA) axis to regulate endotoxin sensitivity (Fig. 5) [32]. Others
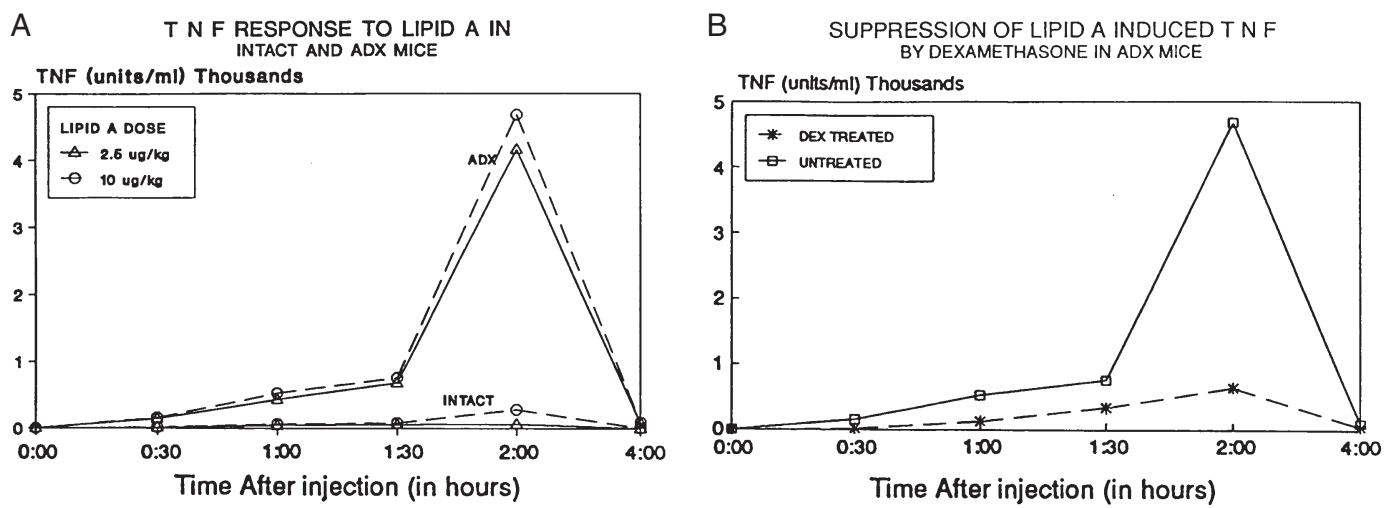

Fig. 5. Adrenalectomy sensitizes mice to lipid A. Adrenalectomy (ADX) increases the sensitivity of mice to lipid A (LA) approximately 500 times. Serum tumor necrosis factor (TNF) is increased 60 times in ADX animals within 2 hours after LA administration. B. Pretreatment of ADX mice with dexamethasone (DEX) suppresses TNF production to normal and restores normal sensitivity of the animals to the lethal effect of LA.Modified from reference [29]. 
also showed that Hypox and ADX rats died within $18 \mathrm{~h}$ after IL-1 $\beta$ infusion (0.5 microg/day) [33].

\section{Endotoxin shock/acute febrile illness/acute phase response $(A P R)$}

APR is induced by immune-derived cytokines, primarily IL-1, IL-6, TNF- $\alpha$, and GM-CSF, which stimulate the hypothalamic paraventricular nucleus (PVN) to release corticotropin releasing hormone (CRH), and vasopressin (VP). The cytokines and these hypothalamic hormones elicit major neuroendocrine and metabolic changes as well as induce immunoconversion in the host organism [21, 34, 35].

Mild infection or sublethal dose of endotoxin elicit a brief elevation of growth hormone (GH) and prolactin (PRL) in the serum. These hormones are proinflammatory and immunostimulatory, so temporarily ADIM is amplified peripherally in the host. In severe trauma, sepsis and shock, GH and PRL are suppressed, whereas glucocorticoids and catecholamines are elevated. Fever and catabolism prevails. The CNS is activated, and the synthesis of acute phase proteins in the liver, cell proliferation in the bone marrow, and protein synthesis by leukocytes are elevated. This is an emergency reaction to save the host organism after the adaptive immune system has failed to contain and eliminate the infectious/noxious agent. During sepsis and endotoxin shock the systemic activation of the complement system and of leukocyte-derived enzymes, tissue-derived brake-down products and highly toxic cytokines seriously threaten survival. Glucocorticoids (GC) regulate pro-inflammatory cytokine production and potentiate the secretion of acute phase proteins by the liver into the serum. Some of these proteins, such as CRP, LBP and mannose binding protein are designed to combine with microorganisms and trigger their destruction by the activation of the complement system and of phagocytes. The increased production of some complement components also helps host resistance. The rise in serum fibrinogen promotes blood clotting, which can serve to isolate the invading agent by triggering thrombosis in infected tissues. A number of enzyme inhibitors are produced as acute phase proteins, which are likely to serve to curb the nonspecific damage inflicted by enzymes released from activated phagocytes and from damaged cells. Catecholamines (CAT) are elevated, which serve to inhibit inflammatory responses and to promote, even initiate, the acute phase response. Serum leptin is also increased, which governs energy metabolism and it is a major stimulator of the immune system (Table 3) [21, 34, 35].
Table 3

Major neuroendocrine changes induced by endotoxins

\begin{tabular}{lccccc}
\hline HPT and & Response & $\begin{array}{c}\text { HPA } \\
\text { axis }\end{array}$ & Response & $\begin{array}{c}\text { Sex } \\
\text { hormone }\end{array}$ & Response \\
\hline TRH & $\downarrow$ & CRF & $\uparrow$ & LH & $\uparrow \downarrow$ \\
TSH & $\downarrow$ & AVP & $\uparrow$ & FSH & $\downarrow$ \\
T4 & $\downarrow$ & ACTH & $\uparrow$ & E2 & $\uparrow \downarrow$ \\
T3 & $\downarrow$ & GC & $\uparrow$ & TS & $\uparrow \downarrow$ \\
PRL & $\uparrow \downarrow$ & $\alpha$ MSH & $\uparrow$ & DHEA & $\downarrow ?$ \\
GH & $\uparrow \downarrow$ & BEND & $\uparrow$ & PS & $\uparrow \downarrow$ \\
IGF-1 & $\downarrow$ & CAT & $\uparrow$ & & \\
INS & $\uparrow$ & & & & \\
GLU & $\uparrow$ & & & & \\
LEP & $\uparrow$ & & & & \\
\hline
\end{tabular}

HPT = hypothalamus pituitary thyroid axis; GLH=growth and lactogenic hormones; $\mathrm{TRH}=$ thyrotropin releasing hormone; $\mathrm{TSH}=$ thyroid stimulating hormone; T3=tri-iodo-thironine; $\mathrm{T} 4=$ thyroxin; $\mathrm{PRL}=$ prolactin; $\mathrm{GH}=$ growth hormone; IGF$\mathrm{I}=$ insulin-like growth factor-I; INS = insulin; GLU = glucagon; LEP = leptin; $\quad \mathrm{HPA}=$ hypothalamus-pituitary-adrenal; $\quad \mathrm{CRF}=$ corticotrophin releasing factor; $\mathrm{AVP}=$ arginine vasopressin; $\mathrm{ACTH}=$ adrenocorticotropic hormone; $\mathrm{GC}=$ glucocorticoid; $\alpha$ $\mathrm{MSH}=\alpha$-melanocyte stimulating hormone; $\beta$-END $=\beta$-endorphin; $\mathrm{CAT}=$ catecholamine; $\mathrm{LH}=$ luteinizing hormone; $\mathrm{FSH}=$ folliculus stimulating hormone; E2 = estradiol; TS = testosterone; DHEA = dehydroepyandrosterone; $\mathrm{PS}=$ progesterone. Modified from reference [19].

If the acute phase reaction fails to protect the host, shock will develop. Patients with subclinical adrenal insufficiency succumb to septic shock almost invariably if glucocorticoid therapy is not given. However, glucocorticoid treatment of septic patients with normal adrenal function has not been helpful. During APR GC and CAT suppresses the T-cell regulated adaptive immune function by stimulating suppressor/regulatory $\mathrm{T}$ lymphocytes and amplify natural immune mechanisms several hundred to a thousand times within 24-48 hours. This phenomenon has been designated as immunoconversion from adaptive/natural immune function to highly amplified natural immunity. Immunoconversion is initiated by immune derived cytokines, and involves profound neuroendocrine and metabolic changes, all in the interest of host defence. Thus, natural immunity is essential for a first and last line of defence and the neuroendocrine system is an important promoter and regulator of this fundamental form of immune defence (Table 3) [21, 33, 35].

\section{Healing}

In most cases APR will proceed to healing. However, in severe cases it may lead to death. Vasopressin is the dominant hypothalamic regulatory hormone during chronic inflammatory conditions, rather than CRH. 
VP stimulates both PRL and the HPA axis and thus it is capable to maintain both the ADIM and INIM systems in homeostasis and harmony. Indeed, our experiments revealed that VP maintains adaptive immunocompetence. On this basis it has been suggested that healing process is under hypothalamic control by VP as the cental regulatory hormone (Table 4$)[34,35]$.

\section{Endotoxin tolerance}

The question was posed whether or not endotoxin tolerance could be used for the prevention of endotoxin shock and of shock due to other causes. During endotoxin tolerance no lysosomal membranes are damaged even if LPS was given repeatedly [8], and the animals survived the endotoxin challenge [9]. However, the use of endotoxin for preventive treatment has been limited by the excessive LPS sensitivity of man and higher animals. For this reason, numerous attempts (using physical, chemical and immunological approaches) were made for the production of LPS preparations that would maintain their ability to induce tolerance, increase natural resistance and maintain its adjuvant property and necrotic effect on tumors, while its toxicity would be decreased or eliminated. Methylation [36] and monophosphoryl lipid A [37-39] have been successful in detoxifying LPS. The capacity to induce natural immunity/resistance was best preserved when ionizing radiation $\left({ }^{60} \mathrm{Co}\right.$-gamma) has been used for detoxification [40-43].

\section{LPS as immunological adjuvant}

Bacterial endotoxins are excellent immunological adjuvants. However, the use of endotoxin in vaccine production is limited due to the undesirable side effects (fever and local inflammation). Nevertheless, the efficacy of some combination vaccines was improved by the small amount of LPS present. RD-LPS maintains its adjuvant potential under various conditions [43-47]. The adjuvant effect of RD-LPS could also be demonstrated by thymidine incorporation in human white blood cells treated with phytohemagglutinin [44]. For this reason it is possible that RD-LPS would be useful also for the potentiation of viral antigens. Inactivated viral vaccines were used in order to prevent the reversion of attenuated viruses to pathogenic strains. However, inactivated viruses are less immunogenic, and require the use of adjuvants for the production of acceptable vaccines. Good adjuvants are in short supply. This warranted the trial of RD-LPS for the potentiation of viral vaccines. We produced a vac- cine against foot and mouth disease with RD-LPS, which was superior to the vaccine in use when tested in mice and pigs. We also succeeded in the production of vaccines against equine and human influenza viruses, which were significantly better than the ones in use at present. RD-LPS adjuvant also increased innate immunity in the vaccinated animals that lasts up to 1-3 weeks. Therefore, it will provide protection prior to the induction of adaptive immunity [45, 49,50].

\section{EXPERIMENTAL THERAPY WITH RD-LPS}

Newborn mice become more susceptible to the lymphocytic choriomeningitis virus if treated with LPS or RD-LPS. This is related to the immunoadjuvant effect of LPS/RD-LPS [47]. Ionizing radiation does not affect the stimulation of the lymphoid system by LPS $[50,51]$. A vaccine was produced against human influenza virus [49]. Similar results were obtained in experiments with Hemophilus influenzae [53, 54].

Newborn germ-free miniature piglets treated with a single dose of RD-LPS showed a fully developed lymphoid system in a short period (10-14 days), which was comparable histologically to the immune system of conventional animals of similar age. Untreated germfree animals had an atrophic immune system [45]. It is likely that natural resistance, or rather its decrease, plays and important role in tumor progression. It is known that killed bacterial cultures [55] and bacterial endotoxin exert antitumor activity [37]. It is unfortunate that the use of LPS is limited in this situation by the harmful side effects it may have. One of the most important cytokines induced by LPS is TNF, which has been tested for tumor therapy with great expectations. Unfortunately, TNF is responsible in part for LPS toxicity. Thus, the toxic effect is related to the antitumor activity. This assumption is supported by the observation that it is possible to inhibit most of the harmful effects of LPS by anti-TNF monoclonal antibodies. One should note, however, that a chemically detoxified toxin preparation, and monophosphoryl lipid A, could exert some antitumor effect [37].

It appears to be very important that the LPS preparations can enhance natural resistance [2, 3, 5]. It is of common knowledge that radiation treatment, chemotherapeutic agents, radiation sensitizing agents, steroids, local heat treatment and even surgical procedures induce a profound decrease in natural resistance. For this reason, the patients become sensitive to infectious agents and a significant proportion fall victims to sepsis caused by Gram-negative bacteria, become 
endotoxemic or develop pneumonia. Anticancer agents as well as ionizing radiation are harmful not only to tumor cells but also to other cells, especially to the membranes. For instance, formyl-leurosine (Kőbányai Gyógyszerárugyár, Budapest), which was effective in animal experiments, could not be used on patients because of cardiotoxicity. It causes hypotonia and membrane damage of cardiac muscle. For this reason we tested the effect of RD-LPS pretreatment on formylleurosine 3 cardiotoxicity. It has been observed in dogs that RD-LPS pretreatment has a minimal effect on the hypotensive effect of formyl-leurosine; however, after $30 \mathrm{~min}$ blood pressure and cardiac function return to normal. Examination of the cardiac muscle of RD-LPS treated dogs did not show the pathological changes of membrane injury, which was present in formylleurosine treated animals $[53,55]$. This experiment indicates that treatment with RD-LPS may protect against the side effects of chemotherapeutic agents and against radiation disease.

Most of the desirable effects of LPS are preserved in RD-LPS: Stimulation of phagocytosis and macrophage activity, elevation of natural antibody and properdin levels. RD-LPS activates the entire immune system, including innate, cellular and humoral immunity. This is the reason for the effectiveness of RD-LPS against diverse noxious agents [9]. It is possible that RDLPS will gain application in complex therapeutic approaches to neoplasia.

Endotoxin affects bone marrow function [49]. Numerous drugs (such as chemotherapeutic agents) and medical procedures (radiation therapy) impair the host defense system. Damage to the bone marrow and lymphatic system leads to the decrease of innate immune resistance. The number of stem cells will decrease in the bone marrow, which leads to a decreased production of white blood cells (especially granulocytes) and agranulocytosis will develop.

The RD-LPS treatment of rabbits can improve or restore bone marrow function. When the animals are given immunosuppressive agents (Immuran, hydrocortisone). Comparative blood counts indicate that RD-LPS increases significantly the production of white blood cells in healthy animals, and it restores almost completely the bone marrow function in Immuran - and hydrocortinose - treated rabbits [45].

\section{CLINICAL OBSERVATIONS}

Experiments described below were done with the permission of the Hungarian Ministry of Health using human volunteers. The first experiment included five subjects (four male and one female). It was observed that RD-LPS given at the dosage level of $7 \mu \mathrm{g} / \mathrm{kg}$ ( $1 \mathrm{mg} / \mathrm{ml}$ solution was used) subcutaneously caused no adverse effects (circulatory, respiratory, urinary tract, cardiac function, nausea, vomiting or diarrhea) within the 24-h observation period. A moderate febrile reaction, an increase in blood pressure and some swelling at the injection site were present. These symptoms significantly diminished or disappeared completely within the next 24-h observation period. The repeated laboratory testing revealed no changes in blood coagulation, liver and kidney function. In contrast, the C3 complement component was elevated in the blood and at $24 \mathrm{~h}$ so was the leukocyte count. It may be concluded, that the dose applied does not elicit a greater reaction than vaccines do, and may be considered harmless and suitable for further clinical studies [57-59].

For the second experiment, 40 volunteers were recruited from university students. We observed that RD-LPS administered subcutaneously at a dose of $4 \mu \mathrm{g} / \mathrm{kg}$ did not produce any clinical symptoms (circulatory, respiratory, urinary, cardiac damage, nausea or diarrhea) at all. At various times after application there was a moderate elevation in body temperature with a maximum of $37.9^{\circ} \mathrm{C}$ in one individual. These symptoms disappeared within $24 \mathrm{~h}$ after application. The laboratory tests performed at $24 \mathrm{~h}$ did not show any alteration in blood coagulation, liver and kidney function. In the serum of treated individuals, taken at $24 \mathrm{~h}$, tumor necrosis factor was elevated. There was also a moderate elevation of serum $\mathrm{T}_{4}$ (thyroxin) in most individuals. The leukocyte count and the $\mathrm{C} 3 \mathrm{com}$ plement fraction in the serum were also increased. The treated individuals were followed for 3 weeks, and two individuals developed a mild form of cold syndrome during that period. It may be concluded that in two experiments TOLERIN was essentially harmless and it caused only a very mild clinical reaction and therefore, it is suitable for further clinical trials [57-59].

After our initial clinical trials had been completed, the Hungarian National Agency of Public Health and Infectious Diseases gave permission for the trial of RD-LPS in nine health institutions in both open and double-blind experiments. During these studies, 110 surgical patients were treated with TOLERIN, and 241 patients receiving conventional medical treatment served as control. The majority of patients had surgery for colon carcinoma. RD-LPS was given to patients $24-48 \mathrm{~h}$ prior to surgery. The results show that in treated individuals there was a decrease of infections and other complications and natural resistance was 
increased, as it was indicated by the laboratory tests (e.g. increase in white blood cell count and C3 complement levels) [57-59].

We also studied testicular carcinoma patients treated with Cysplatin for the correction of granulocytopenia. The experiment was performed on five patients [45]. RD-LPS treatment stimulated bone marrow function, and leukocyte and granulocyte counts increased significantly. This experiment indicates that RD-LPS treatment is useful for the correction of bone marrow suppression caused by chemotherapeutic agents [57-59].

Five individuals suffering from immunodeficiency received RD-LPS, in addition to the usual treatment regimes given to these patients. Although RD-LPS increased CD4 $\mathrm{T}$ cell number in patients with fullblown AIDS, no clinical improvement was obvious in the treated individuals. This observation correlates with our in vitro studies performed earlier. However, in one patient, aged 28 years, there was a significant clinical improvement after the treatment. This patient suffered from severe meningitis and encephalitis of unknown origin, Candida sepsis and of mixed bacterial infections. The CD4+ and CD8+ T-cell count was 250 and 261, respectively. RD-LPS was given two times at $280 \mu \mathrm{g}$ subcutaneously (the second treatment was given 60 days after the first), and it increased significantly the CD4+ and CD8+ T lymphocyte count. There was improvement already after the first treatment and after the second treatment, the CD4+/CD8 ratio was 1.05 . One year later the $\mathrm{CD} 4+\mathrm{CD} 8+$ ratio increased to 2.0 , which is slightly over normal. Bone marrow function was restored by this treatment [57].

Published observations on the mechanisms of action of LPS and RD-LPS and our first clinical trials suggest that RD-LPS may have a wide application in medicine. Further experimental and clinical studies are necessary to elucidate important remaining problems.

\section{CONCLUSIONS}

Fever was known since ancient times as a healing reaction. Bacterial infections that cause fever often exerted beneficial effect on host defense. The isolation of lipopolysaccharide endotoxin from Gram negative bacteria by Boivin initiated scientific investigations on the biological effects of LPS. Endotoxin induced nonspecific resistance to infections, and it stimulated properdin. Repeated small doses of LPS induced endotoxin tolerance, whereby the tolerant animals were able to withstand much higher doses of endotoxin. Moreover, small doses of LPS could prevent infectious diseases.

The immune system may be divided into Adaptive Immunity (ADIM) and Innate resistance (IR) to infections and noxious insults. IR involves mechanisms of Non-Immune resistance and Innate or Natural Immunity (INIM/NIM). Non-immune mechanisms are species specific resistance, various barriers and chemical defense. INIM cells of the immune system generate polyspecific innate immune reactions. Both ADIM and INIM may be divided into cell mediated and humoral immunity. Adaptive immune reactions may be actively developed, or passively transferred by antibodies or immune lymphocytes.

Germ-line genes code for innate immune receptors, which are constant, so such receptors never change and ready to function as soon as they are expressed on the host cells. INIR evolved to recognize evolutionally conserved, cross-reactive (homologous) epitopes (homotopes). Homotopes never change. The Lipid A portion of LPS from Gram negative bacteria is such a homotope, it is expressed in all types of Gram negative organisms, regardless of pathogenecity. The innate immune system evolved to recognize this highly suitable homotope by toll like receptor (TLR)-4. This single receptor is capable of recognizing all strains of Gram negative bacteria and defend the host against them.

LPS has no inherent toxicity, but activate the entire immune system, the endocrine system and indeed the entire organism responds to LPS, because of the wide distribution of TLR in the body. Innate immune defense is never lost, it is the first to provide host defense and is with us until our last moments of life.

Toll like receptors recognize specifically infectious agents, but it is emerging now that TLR participate also in physiology of the host. The CNS the entire immune system and many other tissues and organs express TLR. During acute illness, the entire organism participates in the fight against the infection/injury.

Mast cells are closely associated with sensory nerves, and serve as sensory organs for the CNS for the recognition of antigen penetration in the body. Must cells also transmit to the CNS inflammatory stimuli, as they sense inflammatory cytokines, C3a and C5a complement components, bradykinin, etc. Sensory nerves in the vagus transmit signals about immune/inflammatory evens of visceral organs.

Comparative toxicity of LPS revealed that lover animals, (chicken, frog, fish) are resistant to LPS. Cattle and pig show very high sensitivity, laboratory rodents 
are less sensitive. Today it is clear that during endotoxin shock there is no "toxin", which kills the host, rather, immune -derived cytokines, such as TNF and IL-1 are lethal to the animals administered with endotoxin. Lower animals do not respond violently to endotoxin because their immune system does not recognize, or if so, the system is not capable to produce excess cytokine levels, which would be lethal.

Endotoxin shock or acute febrile illness refers to a condition, which termed scientifically as the acute phase response (APR). APR is an emergency defense reaction induced by acute infection or trauma. Innate immune cells of the monocyte-macrophage series, which are present everywhere in the body, recognize first the insult and secrete the cytokines IL- $1 \beta, \mathrm{TNF}-\alpha$, IL-6 and GM-CSF. These cytokines act on the CNS, the endocrine system and many other tissues and organs in the body. The infectious agents similarly are capable to act on multiple organs and tissues in the body. So during APR the entire organism is mobilized: the CNS, the HPA axis, the sympathetic nervous system are central regulators of APR. The bone marrow, leukocytes and the liver are activated. Natural antibodies increase, and acute phase proteins (APP) in the liver are amplified. The adaptive immune system is suppressed and INIM is rapidly amplified. Fever and catabolism mobilize the energy resources rapidly from the body. The metabolic and energy demand is so high that it can only be satisfied by rapid brake down (catabolism) of bodily resources.

In most cases, APR will subside and vasopressin takes over the regulation of healing and recovery from the disease. VP stimulates both the HPA axis and PRL, so it is capable of establishing homeostatic conditions, which lead to the restoration of normal immune function and healing of the organism.

A major obstacle to the exploitation of the beneficial effects of LPS was toxicity. Methylation and the development of monophosphoryl lipid A have been successful in detoxifying LPS. However, the capacity to induce natural immunity/resistance was best preserved when ionizing radiation $\left({ }^{60} \mathrm{Co}\right.$-gamma) has been used for detoxification of LPS.

Bacterial LPS is an excellent adjuvant. Successful vaccines were produce with RD-LPS to foot and mouth disease of animals and to human influenza virus. These vaccines were superior to the ones used for immunization. During vaccination with RD-LPS Natural Immunity of the animals was also elevated.

RD-LPS inhibited Hemophilus influenzae infections, and radiation disease. It stimulated bone marrow, and lymphoid development in new-born germ free miniature piglets. RD-LPS showed an anti tumor effect and stimulation of TNF production. RD-LPS helped to restore immunocompetence after immunosuppressive insults, such as radiation disease, immunosuppression by cytotoxic drugs, surgery, steroid therapy. In fact RD-LPS activates the entire immune system, including innate, cellular and humoral immunity. It may be useful in complex therapeutic approaches to neoplasia and the stimulation of bone marrow function.

RD -LPS is safe to use in patients and has been approved for human use in Hungary. Initial clinical trials are encouraging.

\section{REFERENCES}

[1] Boivin A, Mesrobeanu J, Mesrobeanu L. Technique pour la preparation des polysaccharides microbiens specifiques. C.R. Soc. Biol. 1933; 113: 490.

[2] Nowotny A. In search of active sites in endotoxins. In: Nowotny A, editor. Beneficial Effects of Endotoxins. New York: Plenum Press; 1983. pp. 1-55.

[3] Wright AE. A short treatise on anti-typhoid inoculation, containing an exhibition of the principle of the method and summary of the results achieved by its application. Westminster, Constable, 1904.

[4] Landy M, Pillemer L. Elevation of properdin levels in mice following administration of bacterial lipopolysaccharides. J. Exp. Med. 1956; 103: 823-830.

[5] Rowley D. Stimulation of natural immunity of Escherichia coli infections. Lancet. 1965; 1: 232-240.

[6] Merétey K. Changes of imunological balance in the mirror of the production of natural antibody (Hungarian), Ph.D. Thesis, Budapest, 1972.

[7] Pillemer L, Blum L, Lepow JH, et al. The properdin system and immunity. Science. 1954; 120: 279-284.

[8] Beeson RB. Tolerance of bacterial pyrogens. J.Exp. Med. 1947; 86: 29-35.

[9] Bertók L, Chow D. Eds. Berczi I, Szentivanyi A. series Eds. Natural Immunity, In Neuroimmune Biology, vol. 6, Elsevier; Amsterdam: 2005.

[10] Rupec RA, Boneberger S, Ruzicka T. What is really in control of skin immunity: lymphocytes, dendritic cells, or keratinocytes? facts and controversies. Clin Dermatol. 2010 Jan-Feb; 28(1): 62-66.

[11] Harvima IT, Nilsson G, Naukkarinen A. Role of mast cells and sensory nerves in skin inflammation. G Ital Dermatol Venereol. 2010; 145(2): 195-204.

[12] Brandtzaeg P. The mucosal immune system and its integration with the mammary glands. J Pediatr. 2010; 156(2 Suppl): S815.

[13] Sabbadini ER, Berczi I. The submandibular gland: a key organ in the neuro-immunoregulatory network? Review. Neuroimmunomodulation. 1995; 2: 184-202.

[14] Redzic Z. Molecular biology of the blood-brain and the blood-cerebrospinal fluid barriers: similarities and differences. Fluids Barriers CNS. 2011; 8(1): 3.

[15] Liggett TE, Balabanov R. Immune Aspects of the BloodBrain Barrier. In The Brain and Host Defense, Neuroimmune biology volume 9. Arnason BA, ed, Berczi I, Szentivany A. Volume eds. Elsevier; Amsterdam: 2010. 
[16] Bonkowski D, Katyshev V, Balabanov RD, Borisov A, DoreDuffy P. The CNS microvascular pericyte: pericyte-astrocyte crosstalk in the regulation of tissue survival. Fluids Barriers CNS. 2011; 8(1): 8 .

[17] Greenwood J, Heasman SJ, Alvarez JI, Prat A, Lyck R, Engelhardt B. Review: leucocyte-endothelial cell crosstalk at the blood-brain barrier: a prerequisite for successful immune cell entry to the brain. Neuropathol Appl Neurobiol. 2011; 37(1): 24-39.

[18] Nagy Z, Berczi I, Bertok L. Experimental data on the pathogenesis of edema disease of swine. Clinical picture, gross and microscopic lesions related to endotoxin shock. Zbl Vet Med Reihe B. 1968; 15: 504-511.

[19] Berczi I, Bertok L, Baintner K, Jr, Veress B. Failure of oral Escherichia coli endotoxin to induce either specific tolerance or toxic syndromes in rats. J Path Bact. 1968; 96: 481-448.

[20] Bertok L. Bile acids in physico chemical host defense. Pathophysiology (Elsevier). 2004; 11: 139-195.

[21] Berczi I, Szentivanyi A. The acute phase response. In Neuroimmmune Biology, Volume 3: The ImmuneNeuroendocrine Circuitry. History and Progress. Berczi I, Szentivanyi A, Editors, Elsevier; Amsterdam: 2003. pp. $463-$ 494.

[22] Berczi I. Neuroendocrine response to endotoxin. Ann NY Acad Sci. 1998; 851: 411-415.

[23] Berczi I. Antigenic recognition by the brain. The brain is an immonocompetent organ. In New Insights to Neurimmune Biology, Berczi I. editor. Elsevier; Amsterdam: 2010. pp. 145 159. Elsevier Insights, www.amazon.com.

[24] Medzhitov R, Janeway CA, Jr. Decoding the patterns of self and nonself by the innate immune system. Science. 2002; 296 : 298-300.

[25] Berczi I, Chow DA, Sabbadini ER. Neuroimmunoregulation and Natural Immunity. Domestic Anim Endocrin. 1998; 15: 273-281.

[26] Flo HN, Aderen A. Pathogen recognition by Toll-like receptors. In Natural Immunity, Neroimmune Biology, volume 5 , Bertok L. Chow DR. eds. Berczi I. Szentivanyi A. Series eds. Elsevier; Amsterdam: 2005. pp. 167-82.

[27] McKay DM, Bienenstock J. The interaction between mast cells and nerves in the gastrointestinal tract. Immunol Today. 1994; 15(11): 533-538.

[28] Berczi I. Integration and regulation of higher organisms by the neuroimmune supersystem. Int. J. Integrative Biol. 2007; 1(3): 216-231.

[29] Berczi I, Quintanar Stephano A, Kovacs K. The brave new world of Neuroimmune Biology. In New Insights to Neurimmune Biology. Berczi I, editor. Amsterdam: Elsevier; 2010; pp. 4-30. Elsevier Insights, www.amazon.com.

[30] Janeway CA, Travers P, Walport M, Slomchick M. Immunobiology. 6th edition. Garland publishing; 2005.

[31] Berczi I, Bertok L, Bereznay T. Comparative studies on the toxicity of Escherichia coli lipopolysaccharide endotoxin in various animal species. Can. J. Microbiol. 1966; 12: 10701071.

[32] Ramachandra RN, Sehon AH, Berczi I. Neuro-hormonal host defence in endotoxin shock. Brain Behav Immun. 1992; 6: 157-116.

[33] van der Meer MJ, Sweep CG, Pesman GJ, Tilders FJ, Hermus AR. Chronic stimulation of the hypothalamus-pituitaryadrenal axis in rats by interleukin 1beta: central and peripheral mechanisms. Cytokine. 1996; 8(12): 910-919.

[34] Berczi I, Quintanar-Stephano A, Kovacs K. Chapter 14. Immunoconversion in the Acute phase response. In Cytokines, Stress and Immunity. Nicholas P Plotnikoff,
Robert E. Faith, Anthony J. Murgo, Robert A. Good, editors. Boca Raton, FL, USA: CRC Press, Taylor \& Francis Group; 2006. pp. 215-254.

[35] Berczi I, Quintanar-Stephano A, Kovacs K. Neuroimmune regulation in immunocompetence, acute illness, and healing. Ann N Y Acad Sci. 2009; 1153: 220-239.

[36] Szilágyi T, Csernyánszky H, Gazdy E, et al. Studies on lysosomal enzyme liberating and endotoxin tolerance inducing capacity of radio-detoxified endotoxin. Acta Microbiol. Acad. Sci. Hung. 1982; 29: 155-159.

[37] Bertók L. Radiodetoxified endotoxin as a potent stimulator of nonspecific resistance. Persp. Biol. Med. 1980; 24: 61-66.

[38] Nowotny A. Endotoxin preparations. Nature. 1963; 197: 220230.

[39] Ribi E, Amano K, Cantrell J, Shwartzman S, Parker R, Takayama K. Preparation and antitumor activity of nontoxic lipid A. Cancer Chemother. Pharmacol. 1982; 12: 91-98.

[40] Toyoda T, Kassell NF, Lee KS. Induction of tolerance against ischemia/reperfusion injury in the rat brain by precondititoning with the endotoxin analog diphosphoryl lipid A. J. Neurosurg. 2000; 92: 435-441.

[41] Xi L. Nitric oxide-dependent mechanism of anti-ischemic myocardial protection induced by monophosphoryl lipid A. Zhongguo Yao Li Xue Bao. 1999; 20: 865-871.

[42] Bertók L, Kocsár L, Várterész V, et al. Method for the production of ionizing radiation induced detoxified bacterial endotoxin preparation (Hungarian). Budapest, Hungarian Patent. 1973; (No. 162,973/-61-k-27/02.23/00).

[43] Previte JJ, Chang Y, El-Bisi HM. Detoxification of Salmonella typhimurium lipopolysaccharide by ionizing radiation. J. Bacteriol. 1967; 93: 1607-1612.

[44] Naidu MD, Chander R. Immunomodulation of macrophages by radio-detoxified lipopolysaccharide of Salmonella typhimurium. Indian J. Exp. Biol. 1999; 37: 283-289.

[45] Nerkar DP, Bandekar JR. Stimulation of macrophages and antitumor activity of radiodetoxified endotoxin. Microbiol. Immunol. 1986; 30: 893-897.

[46] Temesi A, Bertók L, Pellet S. Stimulation of human peripheral lymphocytes with endotoxin and radiodetoxified endotoxin. Acta Microbiol. Acad. Sci. Hung. 1983; 30: 13-17.

[47] Bertók L. Nonspecific resistance: the role of bile acids and bacterial endotoxins (Hungarian), Acta Physiologica (Monographic Series)2nd. Budapest: Scientia; 2002.

[48] Elekes E. Nonspecific stimulation of antibody production in intact and immuno-depressed animals (Hungarian), Ph.D. Thesis: Budapest; 1978.

[49] Merétey K, Elekes E, Bertók L. Antibody production in rats treated with endotoxin. Ann. Immunol. Hung. 1973; 17: 6366.

[50] Elekes E, Bertók L, Merétey K. Adjuvant activity of endotoxin preparations in normal and irradiated rats. Acta Microbiol. Acad. Sci. Hung. 1978; 25: 17-21.

[51] Sólyom F, Bertók L. Application of radio-detoxified endotoxin as adjuvant for experimental foot and mouth disease vaccine. Acta Microbiol. Acad. Sci. Hung. 1985; 32: 233239

[52] Bertók L, Jankovics I. Production of a new, potent human influenza vaccine (Hungarian) Hungarian Patent: Budapest; 2001.

[53] Csukás Zs, Horváth J, Slowok F, Bertók L. Respiratory effect of Haemophilus influenzae endotoxin in mice. Acta Microbiol. Acad. Sci. Hung. 1983; 30: 139-146.

[54] Csukás Zs, Horváth J, Slowik F, Bertók L. Effect of irradiated Haemophilus influenzae endotoxin preparation in mice. Acta Microbiol. Acad. Sci. Hung. 1983; 30: 247-253. 
[55] Juhász-Nagy S, Sótonyi P, Bertók L. Haemodynamic pattern of formyl-leurosine induced acute cardiovascular depression in the dog. Acta Chir. Acad. Sci. Hung. 1985; 26: 35-49.

[56] Bertók L, Juhász-Nagy S, Sótonyi P. Prevention of cardiac damages induced by formly-leurosine. a potent cytostatic agent, by radiodetoxified endotoxin (TOLERIN) in dogs. Immunopharmacology. 1984; 8: 13-17.

[57] Bánhegyi D, Várnai F, Horváth A, Bertók L. Treatment of severe immune deficiency with radio-detoxified endotoxin. J. Med. 1992; 23: 154-156.
[58] Bertok, L. Radio-detoxified endotoxin activates natural immunity: A review. Pathophysiology. 2005; 12: 85-95 (Elsevier).

[59] Bertok, L. New Prospect for the enhancement of natural immunity. In "Natural Immunity", in. "NeuroImmune Biology" vol.5, Bertok L, Chow DA. Ed. Berczi I, Szentivanyi A. Series Ed. Amsterdam; Elsevier: 2005; pp. 289-307. 